\title{
A Stereo Self-adjustment Methodology for Resuming Active Camera Operation
}

\author{
Masafumi Nakagawa, Yoshihiro Kawai, and Fumiaki Tomita \\ National Institute of Advanced Industrial Science and Technology, Japan \\ m.nakagawa@aist.go.jp, y.kawai@aist.go.jp, f.tomita@aist.go.jp
}

\begin{abstract}
A stereo system for vehicles, Unmanned Aerial Vehicles (UAVs), and hand eye systems must tolerate vibration of its cameras and be robust against disturbances. We propose a stereo self-adjustment methodology for resuming autonomous stereo system operations. The methodology aims to achieve autonomous recovery of camera directions, even if the cameras rotate after a weak impact. In autonomous recovery, the self-adjustment procedure calculates the rotation values of rotated cameras. The camera directions of the stereo system then recover their initial directions using the rotation parameters. Moreover, we have verified the validity of our approach through experiments using an active stereo camera in which three cameras can rotate independently.
\end{abstract}

Keywords: Self-adjustment, Maintenance free stereo system, Active camera resume operation.

\section{Introduction}

A stereo vision system for vehicles and Unmanned Aerial Vehicles (UAVs) must tolerate vibration of its cameras. Stereo systems and hand eye systems in an intelligent factory also require high robustness against camera parameter changes during long-term operation.

In general, accurate $3 \mathrm{D}$ measurements using the stereo system require accurate camera parameter values, as calculated via camera calibration, which is normally conducted with a known test pattern board before the 3D measurements begin. However, shock or vibration to the stereo system may cause loss of the camera parameters, preventing accurate 3D measurements.

Tolerance to vibration in the stereo system can be considerably improved by mounting it on a stabilizer, which can reduce the influence of vibration on the cameras [1]. However, an expensive hardware system is required. If the camera parameters are lost during long term operation of the stereo system, they can be recovered via camera calibration using a test pattern board. However, frequent camera calibration is not practical for stereo systems in general environments. Alternatively, selfcalibration, which autonomously estimates the current camera parameters using unknown observation data, can achieve calibration with unknown targets $[2,3,4,5,6]$. Accordingly, we believe that self-calibration has the potential to develop stereo systems that are vibration tolerant. Therefore, self-calibration is an essential technique 
for use with vehicles, UAVs, and hand eye systems. The self-calibration methodology is characterized as follows.

'Self-calibration':

- A process of camera parameter estimation after a strong impact to the stereo camera

- Internal and external camera parameters are unknown.

In general, self-calibration is a flexible technique with few limitations. However, in theory, there are anomalous camera arrangements for which unique parameter estimation is impossible, and self-calibration becomes unstable in the neighborhood of these anomalous camera arrangements. Moreover, independence between parameters can be insufficient, because the available restriction parameters are fewer than the parameters being estimated. Accordingly, self-calibration processing is often unstable from the viewpoint of numerical evaluation, even if the camera arrangement is far from an anomalous camera arrangement [7]. Therefore, we have added some restrictions on using internal and external parameters. The self-adjustment methodology that we have developed is characterized as follows.

'Self-adjustment':

- The process of camera parameter modification after a weak impact to the stereo camera

- Internal and external camera parameters are available using camera calibration data as initial values

- Camera calibration processing is required as a preprocessing operation.

We propose a stereo self-adjustment methodology for stereo system resume operation. This aims to achieve an autonomous recovery of camera directions, even if cameras rotate after a weak impact. In the autonomous recovery, the self-adjustment procedure calculates rotation values for the rotated cameras. The camera directions of the stereo system are then returned to their initial values using the rotation parameters. Moreover, we have verified the validity of our approach through experiments using an active stereo camera in which three cameras can rotate independently.

\section{Methodology}

The cameras in the stereo system recover their initial directions via a self-adjustment procedure, as shown in Fig. 1. First, the stereo camera rotation parameters are acquired via a calibration using a known test pattern. In this state, the stereo system can measure objects correctly. However, after an impact to the stereo camera, the convergence angle of the stereo system changes. In this state, the stereo camera cannot measure objects correctly because stereo camera rotation parameters such as roll, pitch, and yaw are lost.

In that state, corresponding points are obtained from stereo images. In this approach, the corresponding points do not require 3D coordinate values. The camera rotation parameters are then recovered to their initial state via the self-adjustment procedure described in the next section. Using these camera rotation parameters, the stereo system obtains modified stereo rotation parameters, and the stereo system can measure objects again. 
Moreover, relative rotation values of camera directions are obtained by calculating a difference between the stereo camera rotation parameters after camera calibration and after the self-adjustment procedure. The cameras can recover the initial directions physically after these relative rotation values are input to the active stereo camera.

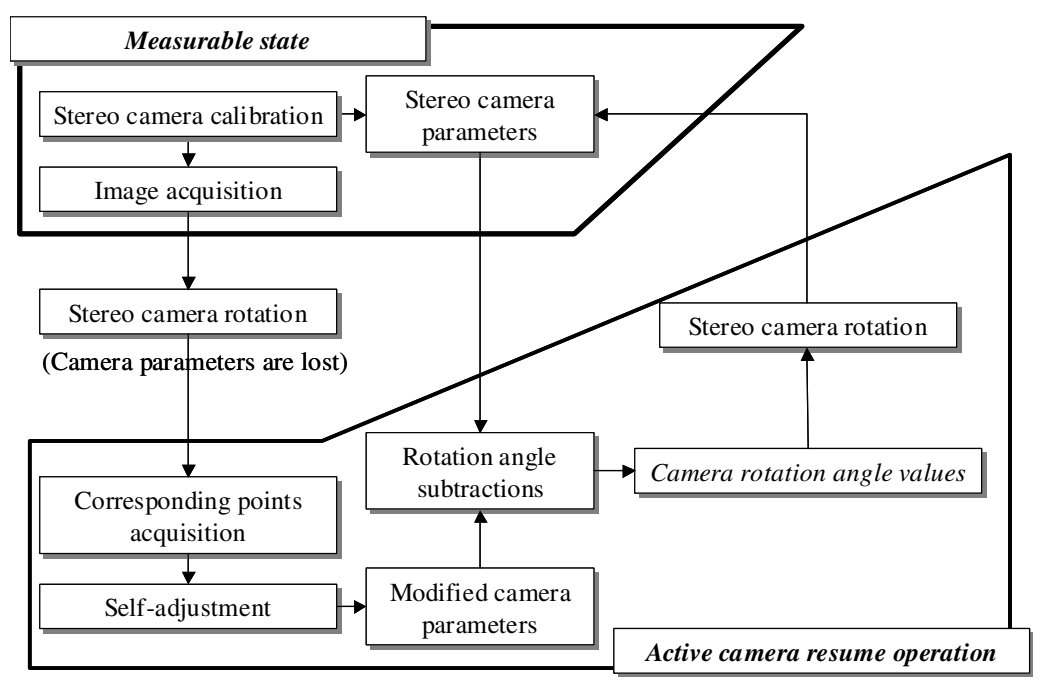

Fig. 1. Processing flow in the actual active camera resume operation

\subsection{Self-adjustment}

We now define the standard camera model as three cameras arranged in a triangle, as shown in Fig. 2. A point in the standard camera image is defined as (x, y). A point changed by camera rotation in a standard camera image is defined as (X, Y). In addition, the rotation parameters for the triplet camera directions are as shown in Fig. 3.

The sum of the position error between $\mathrm{P}_{1}$ and $\mathrm{P}_{c}$, a position error between $\mathrm{P}_{1}$ and $\mathrm{P}_{c}$, and a position error between $\mathrm{P}_{\mathrm{r}}$ and $\mathrm{P}_{\mathrm{c}}$ is minimized in triplet processing. These position errors are obtained as distances from the corresponding point $\mathrm{P}_{1}$ to the

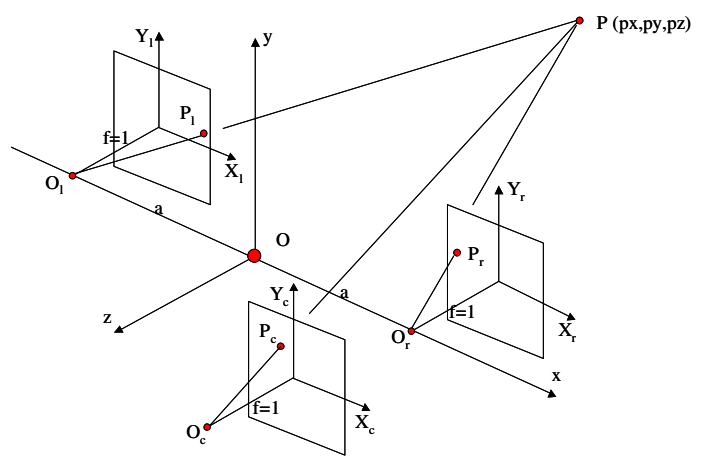

Fig. 2. Standard camera model (triplet) 


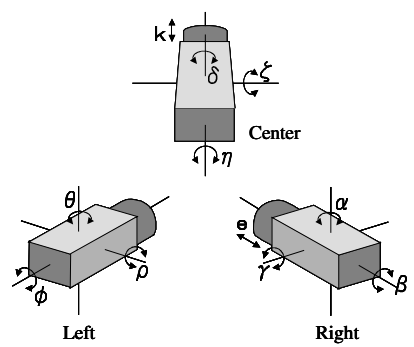

Fig. 3. Rotation parameters (triplet)

epipolar line in the left standard camera image and from the corresponding point $\mathrm{P}_{\mathrm{r}}$ to the epipolar line in the right standard camera image. Therefore, two points on the epipolar line in the left image are acquired.

As shown in Fig. 4, a straight line that connects the focal points of the left and right camera is defined as the $\mathrm{x}$-axis. The center of the two points is defined as the starting point $\mathrm{O}$. Moreover, the values of the focal points of the left camera $\mathrm{O}_{1}$ and center camera $\mathrm{O}_{\mathrm{c}}$ are described as $(-\mathrm{a}, 0,0)$ and $\left(\mathrm{x}_{\mathrm{c}}, \mathrm{y}_{\mathrm{c}}, 0\right)$, When the image coordinate values of $\mathrm{P}_{1}$ and $\mathrm{P}_{\mathrm{c}}$ are described as $\left(\mathrm{X}_{1}, \mathrm{Y}_{1}\right)$ and $\left(\mathrm{X}_{\mathrm{c}}, \mathrm{Y}_{\mathrm{c}}\right)$, a straight line connecting $\mathrm{P}_{\mathrm{c}}$ and $\mathrm{O}_{\mathrm{c}}$ is expressed as $\left(\mathrm{kX}_{\mathrm{c}}+\mathrm{x}_{\mathrm{c}}, \mathrm{kY}_{\mathrm{c}}+\mathrm{y}_{\mathrm{c}},-\mathrm{k}\right)$. Moreover, the focal lengths of the left and right cameras are defined as $\mathrm{f}$. When the straight line is projected into the left image, the track on which the plane $\mathrm{z}=-\mathrm{f}$ intersects with a line that connects $\left(\mathrm{kX}_{\mathrm{c}}+\mathrm{x}_{\mathrm{c}}, \mathrm{kY}_{\mathrm{c}}\right.$ $\left.+\mathrm{y}_{\mathrm{c}},-\mathrm{k}\right)$ and $(-\mathrm{a}, 0,0)$, is expressed as an epipolar line.

$$
\frac{x+a}{k x_{c}-x_{c}+a}=\frac{y}{k Y_{c}+y_{c}}=\frac{z}{-k}
$$

That is, when the value of $\mathrm{z}$ is $-\mathrm{f}$ in the preceding equation, the coordinate values on the epipolar line are expressed as follows.

$$
x=f X_{c}-a+\frac{f}{k}\left(x_{c}+a\right) \quad y=f Y_{c}+\frac{f}{k} y_{c}
$$

Here, points in $\mathrm{k}=\mathrm{f}$ and $\mathrm{k}=\infty$ are defined as $\left(\mathrm{x}_{1}, \mathrm{y}_{1}, \mathrm{z}_{1}\right)$ and $\left(\mathrm{x}_{2}, \mathrm{y}_{2}, \mathrm{z}_{2}\right)$. These points are expressed as follows.

$$
\begin{array}{lll}
x_{1}=f X_{c}+x_{c} & y_{1}=f Y_{c}+y_{c} & z_{1}=-f \\
x_{2}=f X_{c}-a & y_{2}=f Y_{c} & z_{2}=-f
\end{array}
$$

The point $\mathrm{Pl}\left(\mathrm{x}_{0}, \mathrm{y}_{0}, \mathrm{z}_{0}\right)$ and the line that connects $\left(\mathrm{x}_{1}, \mathrm{y}_{1}, \mathrm{z}_{1}\right)$ and $\left(\mathrm{x}_{2}, \mathrm{y}_{2}, \mathrm{z}_{2}\right)$ exist in the same plane orthogonalized to the $\mathrm{z}$-axis, because the coordinate values of point $\mathrm{P}_{1}$ are $\mathrm{x}_{0}=\mathrm{fX}_{1}-\mathrm{a}, \mathrm{y} 0=\mathrm{fY}_{1}$, and $\mathrm{z} 0=-\mathrm{f}_{\mathrm{l}}$. Thus, a quadratic of the position error or the distance value from the point to the line is expressed as follows.

$$
\begin{aligned}
d_{l}{ }^{2} & =\frac{\left\{\left(x_{2}-x_{1}\right)\left(y_{1}-y_{0}\right)-\left(y_{2}-y_{1}\right)\left(x_{1}-x_{0}\right)\right\}^{2}}{\left(x_{2}-x_{1}\right)^{2}+\left(y_{2}-y_{1}\right)^{2}} \\
& =\frac{f^{2}\left\{y_{c}\left(X_{l}-X_{c}\right)-\left(x_{c}+a\right)\left(Y_{l}-Y_{c}\right)\right\}^{2}}{\left\{\left(x_{c}+a\right)^{2}+y_{c}{ }^{2}\right\}}
\end{aligned}
$$


When the focal position $\mathrm{O}_{\mathrm{R}}$ of the right camera is expressed as $(\mathrm{a}, 0,0)$, a quadratic of the position error $d_{r}$ in the right camera is expressed as follows.

$$
d_{r}{ }^{2}=\frac{f^{2}\left\{y_{c}\left(X_{r}-X_{c}\right)-\left(x_{c}-a\right)\left(Y_{r}-Y_{c}\right)\right\}^{2}}{\left\{\left(x_{c}-a\right)^{2}+y_{c}{ }^{2}\right\}}
$$

Therefore, the corresponding points in the three standard camera images after the self-adjustment procedure are expressed in the following equations.

$$
\begin{aligned}
& X_{l}=\frac{x_{l}(\cos \theta \cos \varphi+\sin \theta \sin \varphi \sin \rho)-y_{l}(\cos \theta \sin \varphi-\sin \theta \cos \varphi \sin \rho)-\sin \theta \cos \rho}{x_{l}(\sin \theta \cos \varphi-\cos \theta \sin \varphi \sin \rho)-y_{l}(\sin \theta \sin \varphi+\cos \theta \cos \varphi \sin \rho)+\cos \theta \cos \rho} \\
& Y_{l}=\frac{x_{l} \sin \varphi \cos \rho+y_{l} \cos \varphi \cos \rho+\sin \rho}{x_{l}(\sin \theta \cos \varphi-\cos \theta \sin \varphi \sin \rho)-y_{l}(\sin \theta \sin \varphi+\cos \theta \cos \varphi \sin \rho)+\cos \theta \cos \rho} \\
& X_{r}=\frac{(e+1)\left\{x_{r}(\cos \alpha \cos \beta+\sin \alpha \sin \beta \sin \gamma)-y_{r}(\cos \alpha \sin \beta-\sin \alpha \cos \beta \sin \gamma)-\sin \alpha \cos \gamma\right\}}{x_{r}(\sin \alpha \cos \beta-\cos \alpha \sin \beta \sin \gamma)-y_{r}(\sin \alpha \sin \beta+\cos \alpha \cos \beta \sin \gamma)+\cos \alpha \cos \gamma} \\
& Y_{r}=\frac{(e+1)\left(x_{r} \sin \beta \cos \gamma+y_{r} \cos \beta \cos \gamma+\sin \gamma\right)}{x_{r}(\sin \alpha \cos \beta-\cos \alpha \sin \beta \sin \gamma)-y_{r}(\sin \alpha \sin \beta+\cos \alpha \cos \beta \sin \gamma)+\cos \alpha \cos \gamma} \\
& X_{c}=\frac{(k+1)\left\{x_{c}(\cos \delta \cos \varepsilon+\sin \delta \sin \varepsilon \sin \zeta)-y_{c}(\cos \delta \sin \varepsilon-\sin \delta \cos \varepsilon \sin \zeta)-\sin \delta \cos \zeta\right\}}{x_{c}(\sin \delta \cos \varepsilon-\cos \delta \sin \varepsilon \sin \zeta)-y_{c}(\sin \delta \sin \varepsilon+\cos \delta \cos \varepsilon \sin \zeta)+\cos \delta \cos \zeta} \\
& Y_{c}=\frac{(k+1)\left(x_{c} \sin \varepsilon \cos \zeta+y_{c} \cos \varepsilon \cos \zeta+\sin \zeta\right)}{x_{c}(\sin \delta \cos \varepsilon-\cos \delta \sin \varepsilon \sin \zeta)-y_{c}(\sin \delta \sin \varepsilon+\cos \delta \cos \varepsilon \sin \zeta)+\cos \delta \cos \zeta}
\end{aligned}
$$

Vertical motion is added to the left camera, in addition to the motions when there are two cameras. Moreover, the center or verifying camera has the same degree of freedom as the right camera.

Here, $\sin \theta$ and $\cos \theta$ in the denominators and the numerators in the equations are simplified as $\sin \theta \rightarrow \theta$ and $\cos \theta \rightarrow 1$ to stabilize the solution as follows.

$$
\begin{aligned}
X_{l} & =\frac{x_{l}(1+\theta \varphi \rho)-y_{l}(\varphi-\theta \rho)-\theta}{x_{l}(\theta-\varphi \rho)-y_{l}(\theta \varphi+\rho)+1} & Y_{l} & =\frac{x_{l} \varphi+y_{l}+\rho}{x_{l}(\theta-\varphi \rho)-y_{l}(\theta \varphi+\rho)+1} \\
X_{r} & =\frac{(e+1)\left\{x_{r}(1+\alpha \beta \gamma)-y_{r}(\beta-\alpha \gamma)-\alpha\right\}}{x_{r}(\alpha-\beta \gamma)-y_{r}(\alpha \beta+\gamma)+1} & Y_{r} & =\frac{(e+1)\left(x_{r} \beta+y_{r}+\gamma\right)}{x_{r}(\alpha-\beta \gamma)-y_{r}(\alpha \beta+\gamma)+1} \\
X_{c} & =\frac{(k+1)\left\{x_{c}(1+\delta \varepsilon \zeta)-y_{c}(\varepsilon-\delta \zeta)-\delta\right\}}{x_{c}(\delta-\varepsilon \zeta)-y_{c}(\delta \varepsilon+\zeta)+1} & Y_{c} & =\frac{(k+1)\left(x_{c} \varepsilon+y_{c}+\zeta\right)}{x_{c}(\delta-\varepsilon \zeta)-y_{c}(\delta \varepsilon+\zeta)+1}
\end{aligned}
$$

Moreover, the values of $\mathrm{X}$ and $\mathrm{Y}$ in the preceding equations are assigned to a quadratic of the position error equation. Then, the following equation is minimized with $\mathrm{f}=1$.

$$
S=\sum\left\{\left(Y_{l}-Y_{r}\right)^{2}+d_{l}{ }^{2}+d_{r}{ }^{2}\right\}
$$

Then, the simultaneous equations are solved with matrix $\mathrm{N}$, which has the partial derivatives of $\mathrm{S}$, and matrix $\mathrm{M}$, which has the second-order partial derivatives of $\mathrm{S}$. These equations give $\mathrm{N}_{0}$ and $\mathrm{M}_{0}$ when $\theta=\phi=\alpha=\beta=\gamma=e=0$. Moreover, approximate values of $\theta, \phi, \alpha, \beta, \gamma$, and $e$ are calculated using the following equation. 


$$
M_{0}\left(\begin{array}{l}
\theta \\
\phi \\
\rho \\
\alpha \\
e \\
\delta \\
\eta \\
\zeta \\
\kappa
\end{array}\right)+N_{0}=0
$$

Values of $\left(\mathrm{X}_{1}, \mathrm{Y}_{1}\right),\left(\mathrm{X}_{\mathrm{r}}, \mathrm{Y}_{\mathrm{r}}\right)$, and $\left(\mathrm{X}_{\mathrm{c}}, \mathrm{Y}_{\mathrm{c}}\right)$ are calculated using values obtained from the preceding equation. Then, the values $\left(\mathrm{X}_{1}, \mathrm{Y}_{1}\right),\left(\mathrm{X}_{\mathrm{r}}, \mathrm{Y}_{\mathrm{r}}\right)$, and $\left(\mathrm{X}_{\mathrm{c}}, \mathrm{Y}_{\mathrm{c}}\right)$ are replaced by $\left(x_{1}, y_{1}\right),\left(x_{r}, y_{r}\right)$, and $\left(x_{c}, y_{c}\right)$ to generate $M_{0}$ and $N_{0}$. The calculation is then repeated. The final rotation angles and zoom magnifications are accumulated as the product of the rotation and zoom matrices in the all the calculations. Thus, the adjustment matrix is obtained after some repetitions of the calculation.

\section{Experiments}

We conducted an experiment to validate our approach that cameras in a stereo system can recover their initial directions. The procedure of the experiment based on our approach shown in Fig. 1 is described as follows.

(1) Stereo camera rotation parameters are obtained via stereo camera calibration.

(2) Camera rotation directions are set as the initial camera directions after acquisition of initial images.

(3) Cameras are rotated independently at the set angle values. That is, we prepare a state that cannot measure objects correctly because stereo camera rotation parameters are lost because of convergence angle changes in the stereo system. In this experiment, each camera rotation value is $1^{\circ}$.

(4) Camera rotation angle values, which are obtained via the self-adjustment procedure, are input into the active camera.

(5) Steps (3) and (4) are repeated, five or 10 times in this experiment.

We prepared a triplet camera for this experiment, comprising a left camera, a right camera, and a verifying camera. The system was mounted on a turn stand with ultrasonic motors, as shown in Fig. 4. The resolution of the ultrasonic motors is $0.015^{\circ}$. The specification of this stereo system is given in Table 1. 


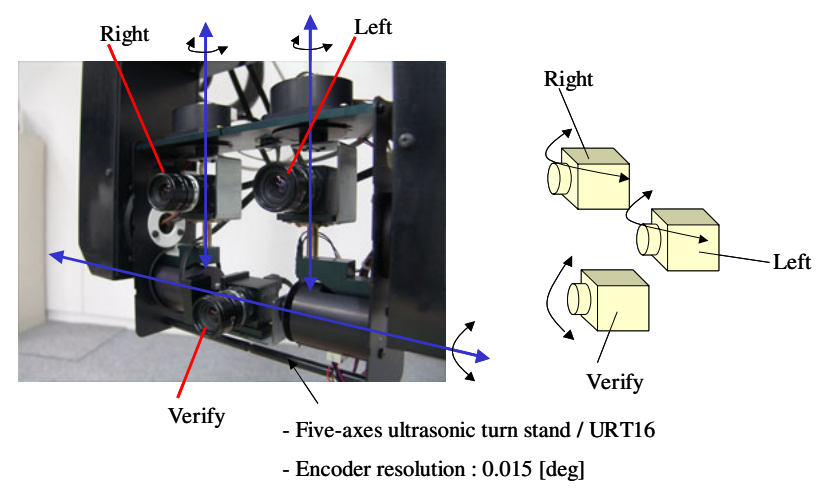

Fig. 4. Triplet camera

Table 1. Specification of stereo system

\begin{tabular}{|c|c|c|c|}
\hline Camera & \multicolumn{3}{|c|}{ Flea (Point Grey Research Inc.) } \\
\hline Lens & \multicolumn{3}{|c|}{$f=16 \mathrm{~mm}$} \\
\hline Image size & \multicolumn{3}{|c|}{ VGA } \\
\hline & \multicolumn{3}{|c|}{ Gray scale } \\
\hline Camera position & $\overline{\text { Left }}$ & Right & $\overline{\text { Verify }}$ \\
\hline Rotation axis & Horizontal & Horizontal & Vertical \\
\hline Baseline & $10 \mathrm{~cm}$ & & \\
\hline Object distance & $130 \mathrm{~cm}$ & & \\
\hline
\end{tabular}

In this self-adjustment experiment, 27 targets were prepared on three boards, with nine targets per board. Images of these boards were captured and general edge detection processing acquired 27 corresponding points automatically. The following camera rotation patterns were prepared to verify the validity of our approach.

- Pattern 1: Left camera rotation

- Pattern 2: Right camera rotation

- Pattern 3: Verify camera rotation

- Pattern 4: Left and right camera rotations

- Pattern 5: Left and verify camera rotations

- Pattern 6: Right and verify camera rotations

- $\quad$ Pattern 7: Right, left, and verify camera rotations.

\section{Results and Discussion}

Results of camera rotation value transitions from patterns 1-7 are shown in Figs 5-11. The vertical axis shows the camera rotation value. Angle value $0^{\circ}$ indicates the initial direction of a camera. The horizontal axis shows a camera rotation event as 'ID'. ID = 0 indicates the angle value to which the camera is first rotated physically to create a state that cannot measure objects correctly because the stereo camera rotation 
parameters are lost because of convergence angle changes in the stereo system. ID values greater than 1 indicate relative rotation values to which the camera rotates physically close to the initial direction via the self-adjustment procedure. Here, rotations leftward or upward are defined as positive values.

The results in all patterns show that camera directions recover the angle values of $0.000 \pm 0.015^{\circ}$, usually within five iterations. The resolution of the active camera rotations is $0.015^{\circ}$ in these experiments, so that the cameras recover the initial directions within the resolution value. Therefore, we can conclude that the results satisfy the required accuracy.

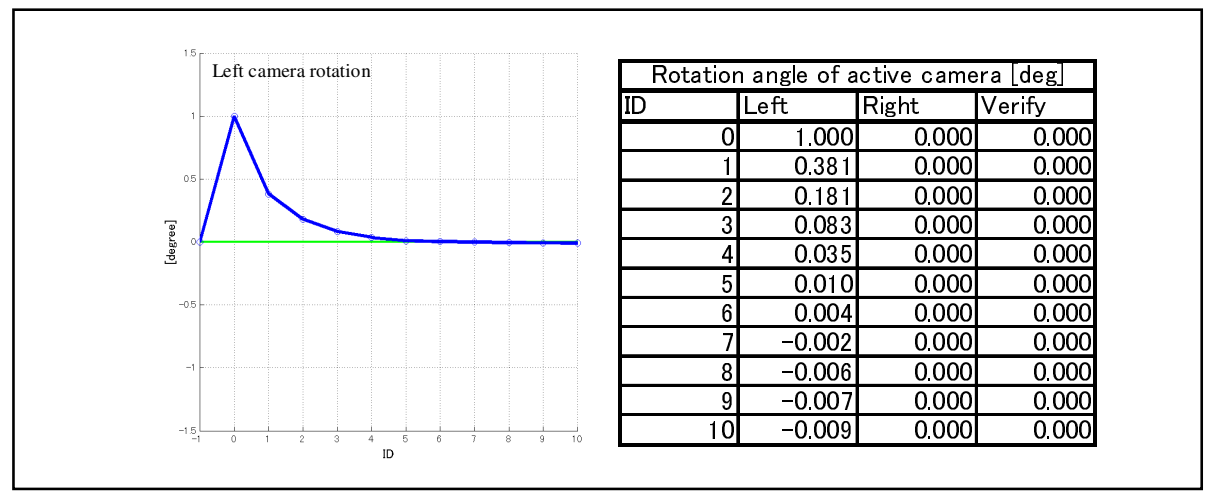

Fig. 5. Pattern 1: left camera rotation

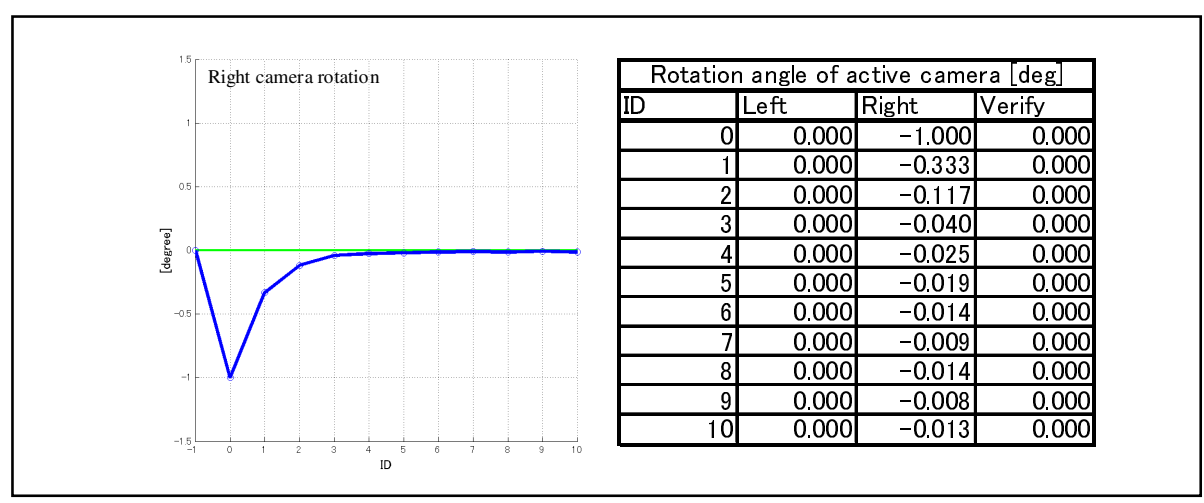

Fig. 6. Pattern 2: right camera rotation

In pattern 7, in which all cameras rotate, the self-adjustment procedure failed to recover the initial camera directions because the base directions of all cameras change. However, the stereo system can still measure objects correctly because the epipolar line is recovered in the self-adjustment procedure. Therefore, an epipolar line recovery, which is a state allowing objects to be measured correctly, is confirmed in pattern 7 . 


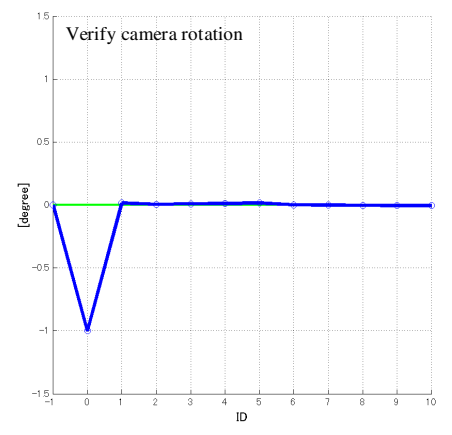

\begin{tabular}{|r|r|r|r|}
\hline \multicolumn{4}{|c|}{ Rotation angle of active camera [deg] } \\
\hline ID & Left & Right & Verify \\
\hline 0 & 0.000 & 0.000 & -1.000 \\
\hline 1 & 0.000 & 0.000 & 0.015 \\
\hline 2 & 0.000 & 0.000 & 0.006 \\
\hline 3 & 0.000 & 0.000 & 0.009 \\
\hline 4 & 0.000 & 0.000 & 0.012 \\
\hline 5 & 0.000 & 0.000 & 0.016 \\
\hline 6 & 0.000 & 0.000 & 0.000 \\
\hline 7 & 0.000 & 0.000 & -0.001 \\
\hline 8 & 0.000 & 0.000 & -0.003 \\
\hline 9 & 0.000 & 0.000 & -0.005 \\
\hline 10 & 0.000 & 0.000 & -0.006 \\
\hline
\end{tabular}

Fig. 7. Pattern 3: verify camera rotation

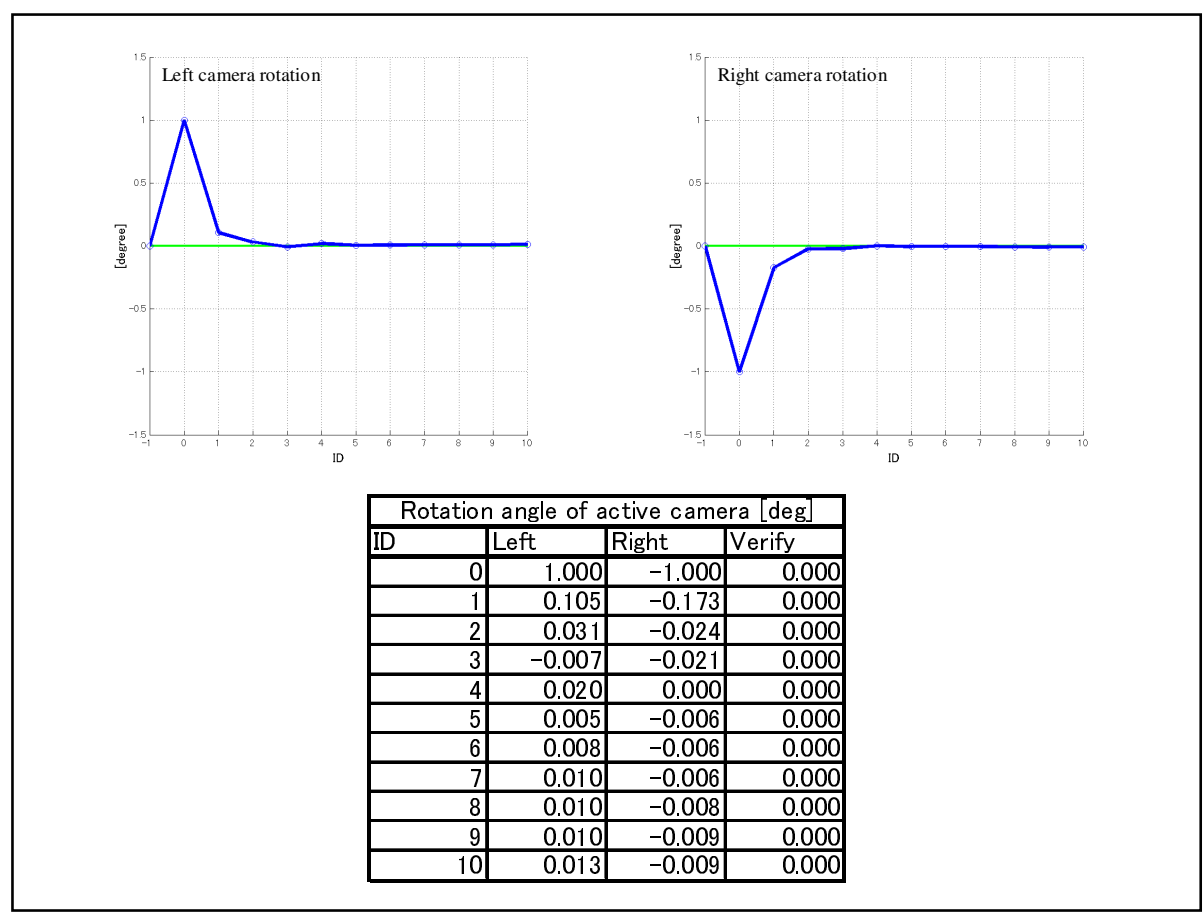

Fig. 8. Pattern 4: left and right camera rotations

Figure 12 shows an epipolar line modification result via the self-adjustment procedure in pattern 7 in which all cameras rotate. The result is transformed based on the standard camera model as shown in Fig. 2. 

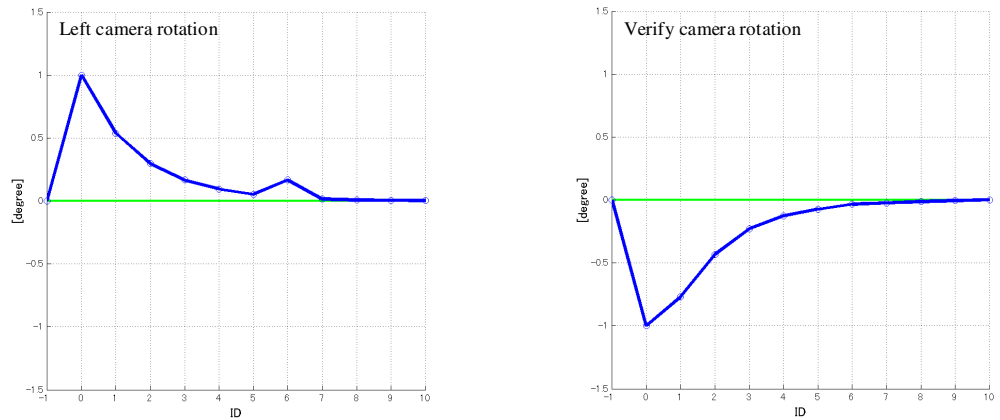

\begin{tabular}{|r|r|r|r|}
\hline \multicolumn{4}{|c|}{ Rotation angle of active camera [deg] } \\
\hline ID & \multicolumn{1}{|l|}{ Left } & Right & Verify \\
\hline 0 & 1.000 & 0.000 & -1.000 \\
\hline 1 & 0.538 & 0.000 & -0.769 \\
\hline 2 & 0.297 & 0.000 & -0.433 \\
\hline 3 & 0.166 & 0.000 & -0.229 \\
\hline 4 & 0.094 & 0.000 & -0.125 \\
\hline 5 & 0.053 & 0.000 & -0.075 \\
\hline 6 & 0.166 & 0.000 & -0.034 \\
\hline 7 & 0.015 & 0.000 & -0.023 \\
\hline 8 & 0.007 & 0.000 & -0.014 \\
\hline 9 & 0.005 & 0.000 & -0.006 \\
\hline 10 & 0.003 & 0.000 & 0.001 \\
\hline
\end{tabular}

Fig. 9. Pattern 5: left and verify camera rotations
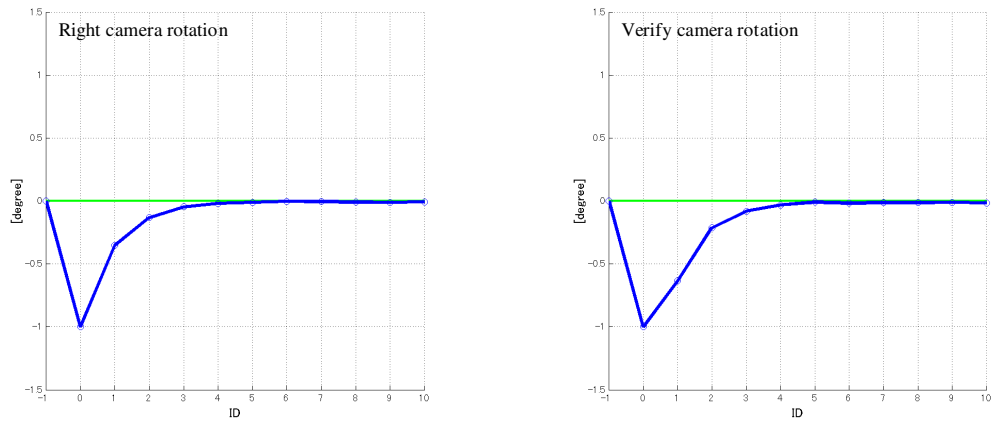

\begin{tabular}{|r|r|r|r|}
\hline \multicolumn{4}{|c|}{ Rotation angle of active camera [deg] } \\
\hline ID & Left & Right & Verify \\
\hline 0 & -1.000 & -1.000 & 0.000 \\
\hline 1 & -0.352 & -0.636 & 0.000 \\
\hline 2 & -0.132 & -0.213 & 0.000 \\
\hline 3 & -0.046 & -0.082 & 0.000 \\
\hline 4 & -0.019 & -0.032 & 0.000 \\
\hline 5 & -0.011 & -0.010 & 0.000 \\
\hline 6 & -0.003 & -0.018 & 0.000 \\
\hline 7 & -0.006 & -0.014 & 0.000 \\
\hline 8 & -0.009 & -0.013 & 0.000 \\
\hline 9 & -0.012 & -0.012 & 0.000 \\
\hline 10 & -0.008 & -0.016 & 0.000 \\
\hline
\end{tabular}

Fig. 10. Pattern 6: right and verify camera rotations 


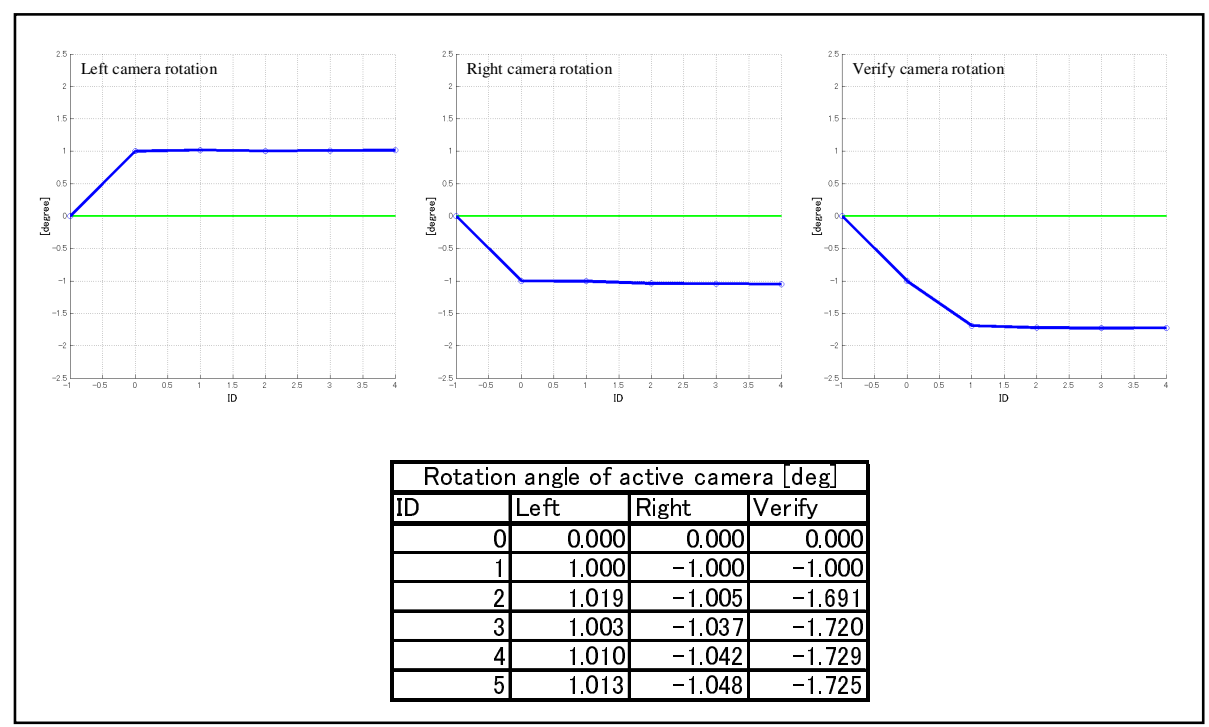

Fig. 11. Pattern 7: all camera rotations
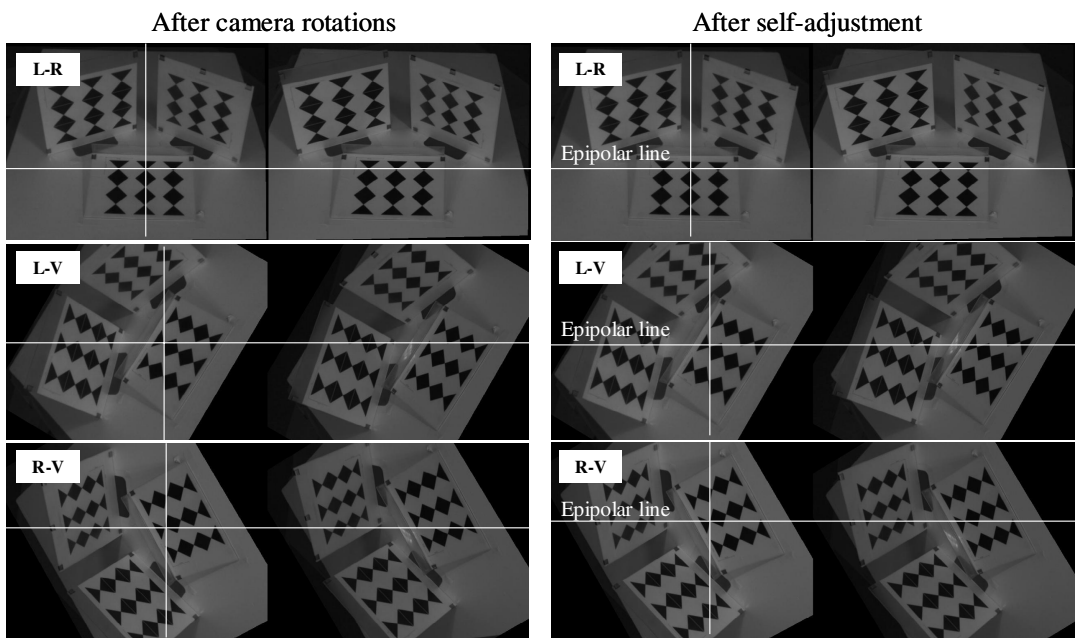

Fig. 12. Epipolar line modification result via the self-adjustment procedure

The epipolar line in a combination of the left and right images after the initial camera rotation, almost passes through corresponding points because the left and right cameras rotate in parallel to the epipolar line. On the other hand, the result shows that the epipolar line in a combination of the left and verify images and the epipolar line in a combination of the right and verify images, do not pass through corresponding points. 
However, the result shows that epipolar lines in all combinations pass through corresponding points. We can thus conclude that the camera rotation directions recover their initial state because the camera rotation parameters are modified via the selfadjustment procedure.

\section{Conclusion}

We have proposed a stereo self-adjustment methodology for resuming stereo system operation. Moreover, we have verified the validity of our approach through experiments using an active stereo camera, showing that the three cameras can rotate independently.

We have confirmed that the self-adjustment procedure calculates the rotation values of rotated cameras. Moreover, the cameras in the stereo system recover their initial directions using the rotation parameters. Camera directions in some camera rotation patterns usually recover the required camera directions within five iterations. Moreover, even when all cameras rotate, the self-adjustment procedure can recover the initial state. From these results, we can conclude that we have successfully achieved the autonomous recovery of camera directions to improve the tolerance or robustness to vibration of stereo system for vehicles, UAVs, and hand eye systems.

\section{References}

1. Gruen, A., Zhang, L.: Sensor modelling for aerial mobile mapping with Three-line Scanner (TLS) imagery Symposium of Commission II, Integrated System for Spatial Data Production, Custodian and Decision Support", Xian, China, 20-23 (August 2002)

2. Hemayed, E.E.: A survey of camera self-calibration. In: Proceedings. IEEE Conference on Advanced Video and Signal Based Surveillance, pp. 351-357 (2003)

3. Armstrong, M., Zisserman, A., Hartley, R.I.: Self-Calibration from Image Triplets. In: ECCV 1996, pp. I:1-I:16 (1996)

4. Kim, H., Hong, K.S.: Practical self-calibration of pan-tilt cameras. IEEE Proceedings on Vision, Image and Signal Processing 148(5), 349-355 (2001)

5. Oliensis, J.: Fast and accurate self-calibration, NEC Res. Inst. In: ICCV 1999, Princeton, NJ, pp. 745-752 (1999)

6. Faugeras, O., Luong, T., Maybank, S.: Camera self-calibration: theory and experiments. In: Sandini, G. (ed.) ECCV 1992. LNCS, vol. 588, pp. 321-334. Springer, Heidelberg (1992)

7. Ueshiba, T., Tomita, F.: National Inst. Advanced Industrial Sci. and Technol., Calibration of Multi - camera Systems Using Planar Patterns. In: CVIM, vol. 2002(102) (CVIM-135), pp. 47-54 (2002) 